\title{
ORGANIZACIÓN DE UN INSTITUTO UNIVERSITARIO DE INVESTIGACIONES CONTABLES
}

\author{
Dr. Mario Biondi* \\ Dr. Mario Wainstein **
}

\begin{abstract}
RESUMEN
Consideramos importante transcribir el presente artículo, publicado en la Revista Contabilidad y Auditoría-diciembre 2001-del Instituto de Investigaciones Contables "Prof. Juan Alberto Arévalo" de la Facultad de Ciencias Económicas de la Universidad de Buenos Aires, Argentina, que nos permite conocer los alcances que tiene un Instituto de Investigaciones Contables, como un estimulo para seguir este ejemplo de desarrollo y satisfacer nuestra inquietante posición de docentes universitarios de la Universidad Nacional Mayor de San Marcos.
\end{abstract}

Además, sus lineamientos y organización pueden aplicarse a las 20 facultades de la UNMSM; y lograr nuestra correcta ubicación en el esquema organizativo institucional vigente de cada Facultad y de la misma universidad. $Y$ en un justo despegue en mayor producción de estudios de investigaciones, libros y publicaciones de artículos científicos para colocar a nuestra Universidad en el sitial que se merece.

Agradecemos a los autores y dignos profesores, colegas nuestros, su posición benevolente, por la transcripción que hacemos en la Revista Quipukamayoc.

\section{LA UNIVERSIDAD. SUS OBJETIVOS}

La universidad contribuye al desarrollo de la cultura mediante los estudios humanistas, la investigación científica y tecnológica y la creación artística. Difunde las ideas, las conquistas de la ciencia y las realizaciones artísticas por la enseñanza y los diversos medios de comunicación de los conocimientos.

La universidad es una comunidad de profesores, alumnos y graduados; forma investigadores originales, profesionales idóneos y profesores de carrera, dispuestos a servir al pais, encauza a los graduados en la enseñanza y en las tareas de la investigación y a través de ellos estrecha su relación con la sociedad.

En esencia, la universidad tiene dos grandes objetivos:

1) La formación de profesionales $y$

2) La formación de investigadores.

* Director del Instituto de Investigaciones Contables «Prof. Juan Alberto Arévalo»-FCE- UBABuenos Aires.

** Director del Centro de Investigación de Auditoría el Instituto de Investigaciones Contables "Prof. Juan Alberto Arévalo"- FCE-UBA-Buenos Aires. 
La investigación es una actividad normal inherente a la condición de docente universitario. (Estatuto Universitario-Universidad de Buenos Aires-Año 1966).

\section{LA INVESTIGACIÓN}

El perfil de un graduado en ciencias económicas requiere, entre otros aspectos, la capacidad para integrarse en grupos interdisciplinarios para desarrollar actividades profesionales, de docencia e investigación. Asimismo debe desarrollar la capacidad para investigar en el área de su especialidad.

La investigación es una actividad normal inherente a la condición de docente universitario.

El instituto es la unidad de investigación. Sus tareas de enseñanza son:

- formar investigadores;

- contribuir a la formación de docentes;

- dirigir a becarios y

- dictar cursos de especialización.

Así, los institutos se crean atendiendo a las necesidades que tengan las Facultades de formar investigadores en determinadas disciplinas que le son propias.

Las condiciones básicas para que funcionen estos institutos son:

- Presencia de especialistas de reconocida capacidad.

- Existencia de medios adecuados que aseguren su funcionamiento regular.

En general, los proyectos de investigación pueden ser de investigación básica (científicos), de investigación aplicada (tecnologías), de desarrollo tecnológico (experimental) o proyectos integrados que contemplen varias de estas etapas.

Los resultados de las diferentes investigaciones están sujetos a diversos grados de incertidumbre lo cual implica que en la realización de proyectos hay también diferentes grados de riesgo en la asignación de recursos para su desarrollo.

La mayor incertidumbre está asociada con los proyectos de investigación básica cuyos resultados son prácticamente imposibles de predecir.

La investigación aplicada contribuye a incrementar la incertidumbre el hecho del desconocimiento acerca de cuánto, quiénes y con qué intensidad adoptarán los resultados del proyecto de investigación.

El hecho de la organización y puesta en funcionamiento de los institutos de investigación generan beneficios aprovechables por la comunidad en su conjunto o por parte de ella, en la medida en que difícilmente se conseguirian sin la implementación de éstos.

Por otra parte, un proyecto de investigación genera beneficios en cuanto a la calificación de los participantes en él, lo cual brinda la posibilidad que personas entrenadas van a poder ser empleadas con ventajas en otros proyectos.

Aún en los casos más desfavorables cuando un proyecto no alcanza los resultados esperados sus beneficios no son nulos ya que la experiencia realizada en los caminos seguidos en la investigación implica una futura economía de esfuerzos y gastos para futuros proyectos. 


\section{CREACIÓN DE INSTITUTO DE INVESTIGACIÓN}

Un instituto de investigación de las Facultades de Ciencias Económicas, tiene las siguientes finalidades.

a) La investigación científica sobre los problemas que corresponden a la ciencia económica y otras disciplinas que le son afines en lo atinente al área que le es pertinente.

b) Colaborar con la docencia universitaria en el más alto nivel.

c) Ejecutar sus trabajos científicos así como asesorar a requerimiento de las autoridades universitarias y también hará lo propio respecto de pedidos de otros organismos públicos y privados.

Para el cumplimiento de sus fines el Instituto de investigaciones tiene las siguientes atribuciones:

a) Investigar sobre temas de la ciencia económica y de otras ciencias y técnicas que están relacionadas por iniciativa del Instituto o por que le han sido propuestos.

b) Celebrar sesiones cientificas para el análisis de temas de su especialidad.

c) Organizar seminarios y comisiones de estudios.

d) Promover la formación de investigadores $y$ proveer la conducente para jerarquizar y facilitar sus trabajos análogamente respecto de los becarios.

e) Dictar cursos y cursillos especiales de profundización referidos a los temas objeto de investigación.

f) Colaborar con las tareas académicas propias de los departamentos de la Facultad, en sus áreas doctorado, carreras $\mathrm{y}$ cursos para graduados $\mathrm{y}$ carrera docente.

g) Facilitar las actividades de los profesores con dedicación especial.

h) Establecer medios permanentes de consulta y de guías de estudio para los alumnos.

i) Mantener relaciones de carácter científico con Instituciones similares del pais y del extranjero.

j) Proponer las publicaciones que resulten expresivas de labor cumplida.

k) Formar el archivo, en la colección ordenada de las informaciones y documentos relacionados con los temas de la especialidad.

1) Organizar la biblioteca especializada, en relación permanente y labor coordinada con la biblioteca de la Facultad.

m) Proyectar y ejecutar trabajos sugeridos por el interés general $y$ otros requeridos por organismos público o privados, de indole científica.

\section{ORGANIZACIÓN DE LOS INSTITU- TOS DE INVESTIGACIÓN CONTABLE}

En cuanto a la organización de los institutos se ha considerado el tratamiento de diversos aspectos, a saber:

\section{a. Creación y Reglamento}

Es necesario que los Institutos de investigación tengan un reglamento que fije las condiciones para su creación y funcionamiento. A modo de ejemplo se desarrolla el siguiente esquema general: 


\section{Introducción}

Se debe referenciar la creación del instituto con el Estatuto Universitario que le debe servir de marco.

Esto requiere definir su misión, por ejemplo:

- Investigación en el campo de las disciplinas en que están dedicados.

- Formación de investigadores.

- Contribución a la formación de docentes.

- Desarrollo de cursos de perfeccionamiento especializado.

\section{Creación}

La base para la creación de un instituto se fija en la realización de trabajos originales que contribuyan al incremento del conocimiento existente.

En este sentido se considera fundamental la formación de investigadores a través de la enseñanza especificamente dirigida a tal efecto.

También puede preverse la creación y funcionamiento de unidades menores de investigación cuando no se dan las condiciones plenas para el funcionamiento de un instituto en forma completa.

\section{Funcionamiento}

Las condiciones recomendables para el funcionamiento adecuado de un instituto de investigaciones son:

- Dirección a cargo de un Profesor de reconocida autoridad y actividad en las tareas de investigación, con régimen de dedicación exclusiva o semiexclusiva.
- Disposición de un adecuado número de investigadores $y$ auxiliares de investigación de dedicación exclusiva y semiexclusiva y de servicios suficientes para asegurar el cumplimiento de las funciones que se le encomiende.

\section{Organización y ubicación}

La organización interna de cada Instituto así como su ubicación dentro de la estructura de la Facultad y su relación con los demás órganos universitarios será establecida en cada caso de acuerdo con las características y exigencias de las tareas y funciones específicas que tiene destinado.

Debe establecerse la dependencia jerárquica del Instituto. Puede depender de un departamento o de una secretaria técnica y eventualmente del decanato.

Los institutos podrian también integrar las cátedras.

Es conveniente que los institutos tengan autonomía científica, estando a su cargo y bajo la exclusiva responsabilidad de sus autoridades la dirección científica $y$, en particular, la formulación de planes de actividad y orientación, evaluación y supervisión de su ejecución.

Anualmente el instituto debe elaborar una memoria o en forma separada, se incluirá un plan de tareas a realizar en el año siguiente.

Se debe establecer las características del presupuesto del instituto por partidas globales o por partidas detalladas. 
Cada instituto deberá dictar su propia reglamentación sobre su funcionamiento, que deberá aprobar el organismo correspondiente.

El Director del instituto será designado por el Consejo Superior de la facultad. En casos excepcionales podrá designarse mediante contrato a plazo fijo, sin requerirse en tales casos la condición de profesor de la Facultad.

El Director del Instituto será responsable de la dirección científica del organismo y de la coordinación de los medios humanos y materiales a disposición.

\section{Personal}

El personal docente del Instituto será designado de acuerdo con las siguientes normas:

- Directamente por autoridad de la Facultad a propuesta del Director del Instituto.

- Por concurso, a pedido del Director del Instituto, cuando se designe personas que no integren hasta el momento parte del personal docente de la Universidad.

- Por contrato, previa propuesta del Director del Instituto.

- Designación interina a propuesta del Director del Instituto.

El personal técnico del instituto será designado de acuerdo con algunas de las siguientes formas, y a propuesta del director del instituto:

- Directamente,

- Por concurso, realizado a propuesta del director

- Por contrato
El personal administrativo y de servicio podrá ser personal estable de la facultad o persona contratada especialmente.

En todos los casos, las designaciones serán hechas por el decano a propuesta del director del instituto.

(Antecedente-Resolución N. ${ }^{\circ} 2853$ de la Universidad de Buenos Aires.)

\section{b. Manual de Organización, Misiones y Funciones}

Un instituto de investigaciones contables debe tener organizada una estructura jerárquica que le permita cumplir con los objetivos trazados.

Se indica a continuación una organización simple de un instituto.

\begin{tabular}{|c|c|c|}
\hline $\begin{array}{c}\text { Secretaria } \\
\text { Administrativa }\end{array}$ & Director & $\begin{array}{c}\text { Secretaria } \\
\text { Técnica }\end{array}$ \\
\hline & $\begin{array}{c}\text { Subdirector } \\
\text { Investigadores } \\
\text { Personal de Apoyo }\end{array}$ & \\
\cline { 2 - 3 } &
\end{tabular}

En los párrafos siguientes se comenta las misiones y funciones para cada uno de los componentes.

\section{Director}

\section{Misión}

Planificar, dirigir, coordinar y evaluar la acción en materia de investigación contable.

\section{Funciones}

1. Mantener las relaciones con las autoridades de la Facultad.

2. Mantener las relaciones con el Instituto similar de las universidades del país y del exterior. 
3. Mantener las relaciones con el Instituto Técnico de Contador Público (ITCP) y con el Centro de Estudios Científicos y Técnicos (CECYT).

4. Aceptación de los planes de investigación de los investigadores del instituto.

5. Elaboración del plan anual de actividades.

6. Elaboración anual de la memoria, donde se describe la acción desarrollada y los proyectos.

7. Control periódico del desarrollo de los planes de investigación.

8. Programación de cursos, seminarios, conferencias y su evaluación.

9. Representación del Instituto en Congresos y otros eventos nacionales y extranjeros.

10. Atención de becarios, doctorandos y futuros investigadores.

11. Supervisar las secretarías técnica y administrativa.

12. Disponer la incorporación y baja del personal con arreglo a las normas administrativas vigentes.

13. Conducción del trámite y subsidios para investigación.

14. Supervisión del apoyo logístico a las tareas de investigación.

15. Disponer las publicaciones del instituto.

\section{Subdirector}

\section{Misión:}

Proponer acciones en materia de investigación contable y actuar como enlace entre la dirección y los investigadores.

\section{Funciones:}

1. Sustituir al Director en representaciones y tareas que éste le asigne.

2. Realizar las tareas encomendadas por el Director del Intituto.

\section{Secretaría Técnica}

\section{Misión:}

Coordinar y ejecutar las directivas del director y del subdirector en materia de investigación contable, apoyando las actividades de los investigadores.

\section{Funciones:}

1. Asistir regularmente a la sede del instituto, responsabilizándose de la atención diaria, desde el punto de vista técnico de acuerdo con el director y subdirector.

2. Mantener los contactos con institutos similares de las universidades del país y del extranjero.

3. Mantener la relación con el Instituto Técnico de Contador Público (ITCP) y con el Centro de Estudios Científicos y Técnicos (CECYT) para los temas técnicos.

4. Supervisión del personal asignado a la Secretaría Técnica y a la Secretaria Administrativa.

5. Apoyo logístico técnico a los investigadores y personal de apoyo a la investigación.

6. Redacción del plan anual de actividades.

7. Seguimiento de los planes de investigación y elaboración de 
informes para la redacción de la Memoria Anual.

8. Organización de los cursos, seminarios y conferencias planificados por la dirección y subdirección.

9. Representar al subdirector en las actividades que se le asignen.

10. Organización de archivos con datos de los docentes del área contable y materias afines.

11. Organización y mantenimiento del archivo técnico; biblioteca y hemeroteca.

12. Gestionar subsidios según directivas de la dirección.

13. Participación en planes de investigación.

14. Concretar las publicaciones que indique la dirección.

\section{Secretaría Administrativa}

\section{Misión:}

Ejecutar las directivas de la dirección en los aspectos administrativos de apoyo a la investigación contable coordinadamente con la Secretaría Técnica.

\section{Funciones:}

1. Asistir diariamente a la sede del instituto, responsablizándose de la atención al público.

2. Atención de la correspondencia $\mathrm{y}$ archivo del Instituto.

3. Atención telefónica de la sede del instituto.

4. Tareas de correspondencia y dactilografía.
5. Mantenimiento del inventario de los elementos de los institutos.

6. Apoyo directo de secretaría para la dirección del instituto.

7. Relación con los servicios de apoyo de la facultad.

\section{Investigadores}

No forman parte de la estructura estable; permanecerán con cargos docentes en el instituto en la medida en que cuenten con planes de investigación en desarrollo.VI. Personal de apoyo a la investigación

(Idem punto VI. Investigadores.)

\section{c. Categorización}

\section{Autoridades:}

Se prevé para la organización de un instituto de investigación contable la incorporación de un director, un subdirector $y$ un secretario técnico designados por el decano con la aprobación del consejo directivo. Integra también la dotación del instituto, personal administrativo e investigadores.

\section{Miembros:}

Además del director, subdirector, secretarios, investigadores y auxiliares, integran el núcleo de recursos humanos los miembros natos.

Los miembros natos son los profesores de la Facultad de las materias que tienen afinidad cn las funciones del instituto.

La designación de los profesores honorarios está reservada a los estudiosos que por su prestigio científico, sus publicaciones y 
actuación en la especialidad han adquirido mérito destacado para tal distinción.

Pueden existir también miembros adscriptos y corresponsales.

\section{d. Planes}

Resulta necesario que el Instituto de Investigaciones tenga un Plan General de Actividades que incluya los proyectos de investigación que se desea desarrollar, cubriendo un período de 2 años.

Anualmente se debe considerar especialmente el Plan de Investigación que debe elevarse a las autoridades de la Facultad, para su correspondiente aprobación.

El Plan General de Actividades deberá cubrir diversos temas a saber:

a) Área Administrativa: Se indicará qué aspectos serán encargados.

b) Área Investigación: Con indicación de los trabajos de investigación que serán encargados.

c) Otras actividades: Incluye la realización de encuentros, cursos, seminarios, conferencias y el tratamiento de temas de actualidad. También puede encargarse reuniones con los profesores de las cátedras correspondientes.

Los Planes de Investigación deben presentarse de acuerdo a un ordenamiento que podría incluir los siguientes datos.

1. Título.

2. Investigador.

3. Aportes previstos.
- Contendrá una breve descripción de los desarrollos que se prevén en el tema.

4. Estado Actual del tema propuesto.

- A efectos ilustrativos se describirá brevemente el estado actual del tema, referenciando la ubicación correspondiente del mismo.

5. Objetivos

- Es importante fijar objetivos que puedan alcanzarse con una descripción clara y sencilla.

6. Plan de trabajo

- Comprende una descripción de las tareas que serán desarrolladas y el tiempo previsto para ello.

7. Vinculación prevista de los resultados con las actividades docentes

- La idea de este punto es establecer una posible relación entre el trabajo que se encarga y su utilización como bibliografía en el futuro.

8. Bibliografía

- Una primera nómina bibliográfica de orientación es conveniente como punto de partida. Lógicamente con la ejecución del trabajo se incorporarán nuevos elementos bibliográficos.

Se ha discutido mucho la necesidad de reformar el enfoque de la investigación. Algunas opiniones encuadran a la investigación de las universidades en un enfoque científico-tecnológico "sobre un modelo de oferta". Otro enfoque es considerar la necesidad de elaborar 
un esquema de politica científica tecnológica desde la "demanda" que posibilite el desarrollo de proyectos de cooperación, sin descuidar la posibilidad de realizar investigaciones de las llamadas "esporádicas".

Con el enfoque de las necesidades de la "demanda" se busca también insertar a la universidad en un mundo competitivo y de excelencia.

La definición del enfoque estratégico de los Planes de Investigación en función de la potencial demanda que los trabajos tengan en el mercado, conforma un novedoso punto de vista que deberá evaluarse. Su puesta en marcha puede representar una nueva fuente de financiamiento con recursos que provengan de trabajos de cooperación y del sector privado.

\section{e. Presupuesto}

El financiamiento de la actividad de un instituto debe instrumentarse con la elaboración de un presupuesto, el cual deberá seguir las pautas incluidas en la planilla anexa y tener en consideración los siguientes aspectos:

1. El presupuesto anual deberá incluir todos los rubros de gastos previstos que correspondan al Instituto en materia de funcionamiento, bibliografia, equipamiento y viajes (internos e internacionales), en su correspondiente especificación.

2. Material de consumo para funcionamiento: importe que se mostrará en la compra de: papeleria, fotocopias, gastos de limpieza, etc.

3. Gastos de viaje. Detalle del importe destinado para gastos de pasajes y viáticos dentro y fuera del país, con la discriminación correspondiente.

4. Gastos fijos. Se agruparán los gastos de conformidad al concepto de parcial.

5. Servicios. Se agruparán los gastos de conformidad al concepto de parcial. Importe a erogar en personal afectado a tareas especificas de actividad cientifica, técnica y/o de apoyo por un breve período. Incluye los contratos por servicios de limpieza, vigilancia, mantenimiento, apoyo administrativo, etc.

6. Inmuebles. Gastos emergentes a los conceptos que se enumeran, remitiendo información detallada de la reparación y construcción.

7. Gastos de mantenimiento. Servicio de reparación de equipamiento administrativo o de apoyo general (mantenimiento de edificio, pintura, pequeños retoques).

8. Otros gastos. Erogaciones no contempladas en ninguno de los rubros de este presupuesto (por ejemplo, la realización de seminarios, congresos, atención de científicos extranjeros, impresión de publicaciones propias, etc.).

9. Bibliografia. Total de gastos de compra de textos, revistas y publicaciones en general y otros directamente vinculados con la actividad de investigación.

10. Equipamiento. Adquisición de equipos. 


\section{RUBROS}

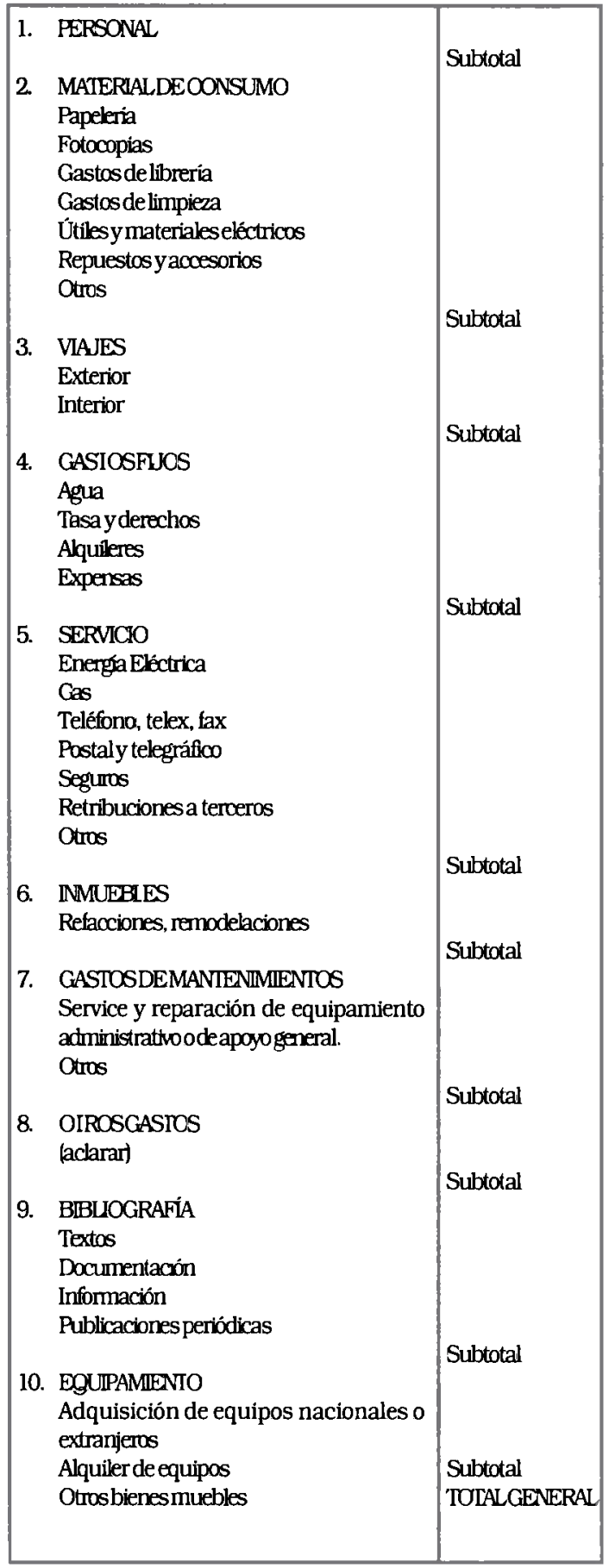

\section{f. Espacio Físico y Equipamiento}

Para la puesta en marcha de un Instituto de Investigación, resulta indispensable contar con un espacio físico y un equipamiento mínimo de oficina.
El espacio físico debe ser suficiente para poder satisfacer las siguientes necesidades:

- Despacho u oficinas privadas 3 (tres) para las oficinas del director, subdirector y secretarias administrativa y técnica.

- Biblioteca y sala de reuniones

- Sala de trabajo

- Lugar para archivo, y depósito de artículos de oficina.

Como equipamiento de oficina se considera el siguiente:

- 1 máquina de escribir.

- 3 computadoras equipadas con sus mesas, impresoras y software, escritorios, sillas y mesas; biblioteca, muebles para archivo.

- 1 linea telefónica con fax incorporado.

- 1 fotocopiadora.

\section{g. Becas y Pasantías}

Bajo esta denominación se incluye las becas internas $y$ pasantias de investigación, que deben tener su correspondiente reglamentación.

\section{Becas Internas:}

La reglamentación debe cubrir los siguientes aspectos:

a. A quienes se destina: a estudiantes y graduados.

b. Características de las becas:

\section{Para estudiantes:}

Becas de introducción a la investigación, como iniciación del estudiante en la investigación cientifica, tecnológica y humanistica, bajo la dirección de un investigador con amplios antecedentes en el tema. 


\section{Para Graduados:}

Becas de iniciación a la investigación y para aquellos graduados universitarios que por los resultados de sus estudios reúnan las condiciones necesarias para iniciar su formación como investigadores mediante la realización de trabajos de investigación bajo la dirección de un investigador con amplios antecedentes en el tema.

Para graduados universitarios que hayan iniciado su formación como investigadores y revelado capacidad para participar en trabajos de investigación, con el fin de continuar dichas tareas y mejorar su formación específica becas de perfeccionamiento en la investigación, bajo la dirección de un investigador formado.

Se puede dar especial consideración a los aspirantes admitidos en planes de doctorados y maestrias o posgrados que requieran trabajos finales de investigación, tesinas o equivalentes.

c. Otorgamiento de becas:

Normativa para la asignación de las becas, de acuerdo con las disposiciones existentes.

d. Aspirantes a Becas: Debe fijarse el procentaje de la carrera cursada, edad límite para el aspirante.

Ejemplo:

- Becas de introducción a la investigación: Estudiantes de la facultad que cursen carreras de grado y que al momento de la presentación del concurso hayan completado el $75 \%$ de la primera carrera de grado, con un máximo de 27 años.

- Becas de iniciación de investigación: Graduados de universidades nacionales $o$ provinciales que hayan cursado carrera de grado y que a la fecha de apertura del concurso tuvieran menos de 5 años de antigüedad en su título de grado y un máximo de 30 años.

- Becas de perfeccionamiento: Hasta 10 años de graduado y hasta 35 años de edad.

También debe contemplarse un régimen de excepciones y el tratamiento de dispensar a graduados de universidades extranjeras cuyos títulos sean homologados por una universidad nacional.

e. Requisitos a cumplir por los aspirantes: Debe considerarse todos los aspectos que se deben cumplir.

f. Duración de las becas: Se considera que 2 años con la posibilidad de prórroga de otro año adicional, conforma el tiempo razonable para la duración de las becas para estudiantes y para graduados.

g. Obligaciones del becario: Debe fijarse un marco general que abarca el cumplimiento de un plan de un trabajo previamente aprobado y el tiempo de dedicación. En el caso de los alumnos becados se considera razonable una dedicación de 20 horas semanales; mientras que las becas para graduados requeririan dedicación exclusiva, incluyendo tareas docentes.

h. Informe sobre el desarrollo de trabajo: en forma periódica y por lo menos una vez al año, los becarios deberán elevar un informe sobre el grado de avance de los trabajos a su cargo. 
i. Directores de becas: Esta tarea deberá ser asignada a profesores de la Facultad debiendo establecerse las tareas de supervisión que deberán ejercer sobre los beneficios de becas de investigación.

\section{Pasantias}

La reglamentación deberá incluir los procedimientos para otorgar becas a estudiantes de grado para pasantías de investigación.

La dirección de este tipo de becas deberá otorgarse a un profesor de la facultad y el becario deberá asignarse a un equipo de investigación.

Se indica a continuación los aspectos que deberán ser considerados en este sistema:

\section{Comisiones de admisibilidad:}

Estudiante de la carrera de grado de la facultad que haya aprobado entre el $50 \%$ y el $75 \%$ de las asignaturas del Plan de Estudios de la Carrera.

Duración de la beca: Podrá fijarse en un periodo de un año con posibilidad de prorrogarse por un año más.

\section{Condiciones a cumplir:}

Debe cubrir los aspectos formales de solicitud de beca, evaluación, calificación asignada al aspirante y sistema de información a cumplir.

\section{h. Sistema de Información}

Un instituto de investigación debe tener un sistema de información interno y externo.

\section{Sistema Interno}

Comprende:

- Comunicaciones internas.
- Citaciones a reuniones.

- Actas o minutas de las reuniones.

- Minutas para el archivo de los eventos que se realicen.

- Comunicaciones de los investigadores sobre el grado de avance de los proyectos.

\section{Sistema Externo}

- Memoria anual.

- Plan de actividades para ejercicio que se indica.

- Presupuesto.

\section{i. Publicaciones}

Todos los trabajos de investigación deberán presentarse en copias suficientes para la provisión de ejemplares a la biblioteca de facultad y remisión a otros centros de investigación.

Resulta importante establecer un mecanismo de evaluación de esos trabajos para lograr su difusión, mediante la publicación de revistas, que edite la propia facultad o en su defecto arbitrar los medios para que el propio instituto de investigación lo edite y lo distribuya.

\section{j. Carpeta de Antecedentes}

La preparación de una carpeta de antecedentes constituye un medio de presentación de un instituto para todos aquellos que se interesan por su existencia y su funcionamiento.

Se expone, a manera de ejemplo, una guía para la confección de una carpeta de antecedentes de un instituto de investigaciones.

$\mathrm{Su}$ objetivo es hacer conocer a terceros las actividades que se desarrollan y además sirve de base de la documentación a presentar en 
caso de solicitar colaboraciones, o realizar convenios con otros entes.

\section{Individualización}

a) Nombre y sigla

b) Sede (dirección completa, teléfono, telex, etc.)

c) Dependencia

d) Estructuras de gobierno y administración

- Director

- Subdirector

- Consejos y asesores u órganos equivalentes

- Comisiones internas

- Organigrama

e) Objetivo y desarrollo

Describir en forma precisa los objetivos específicos que se persigue, si correspondiera, los acontecimientos más significativos que caracterizaron su evolución.

f) Desarrollo de la disciplina en el pais y en la región.

Se hará una semblanza de la situación del desarrollo de la disciplina en el pais y en la región y en especial, cómo se inserta en la misma los temas a desarrollar.

\section{Del Personal}

a) Investigadores

b) Personal profesional

c) Personal técnico

d) Personal administrativo

e) Personal servicios auxiliares

Nómina de agentes con determinación de categoría y Organismo al cual pertenecen:

Agregar como anexo los curriculum del Director y de los investigadores independientes.

\section{Becarios}

a) Internos

b) Externos c) De otros organismos del país

d) De otros paises

(Se detallará nombre y apellido, categoria, tema de beca y director)

\section{Infraestructura}

Proyecto en curso:

Nombre del proyecto

Director

Personal interviniente

Objetivos

Metas alcanzadas o estado de avance

Se confeccionará una hoja por proyecto con una memoria de la investigación de no más de 200 palabras.

\section{Docencia}

Cursos dictados en el último año calendario

Curso regulares

Cursos de posgrado

Coloquios

Seminarios

Tesis realizadas en el último año calendario

a) De licenciatura

- Aprobadas

- En ejecución

b) De doctorado

- Aprobadas

- En ejecución

(Se detallará nombres y apellidos del tesista, tema y director)

\section{Congresos y reuniones cientificas}

Organizados por el Instituto

Participación en eventos dentro del país

Participación en eventos en el exterior 
Otras Actividades

- Distinciones honorarias

- Colaboraciones

- Visitantes del país y del extranjero

- Conferencias

- Otras

\section{Trabajos realizados y publicados}

Trabajos publicados

Trabajos terminados y no enviados todavia para su publicación

Informes y memorias técnicas

Comunicaciones

Libros

Revista-boletín

\section{Convenios en vigencia}

Con universidades

Con empresas

Con otros organismos nacionales

Con organismos extranjeros e internacionales

Se indicará en cada caso la duración del convenio, sus objetivos y la tarea realizada.

\section{Acciones de asesoramiento y servicios tecnicos}

Con universidades

Con empresas

Con otros organismos nacionales

Con organismos extranjeros e internacionales

Se indicará en cada caso la duración del convenio, sus objetivos y la tarea realizada.

\section{Documentación y Biblioteca}

Colecciones de revistas que se reciben en forma continuada

Adquisiciones

Donaciones
Traducciones efectuadas

Servicio de intercambio

\section{Presupuesto Vigente}

Se confeccionará un cuadro resumen del presupuesto del ejercicio vigente.

\section{Desarrollo Previsto}

Se confecionarán memorias descriptivas de las necesidades de desarrollo previstas y el origen de financiamiento.

\section{Construcciones:}

(edificios nuevos, modificaciones de lo existente, ampliación, etc.)

Equipamiento:

Indicar el instrumental a incorporar cada año y su costo individual.

Personal científico a incorporar: Indicar la categoria y año en que se incorporará y si se trata de becarios en el exterior, investigación que regresa al grupo a incorporar, si se cumple el plan propuesto. Acompañar curriculum si es posible.

Capacitación de personal científico:

Becas internas

Becas externas

Asistencia técnica externa

Del pais

Del exterior

Bibliografía

Gastos de funcionamiento

Evaluación de las investigaciones ya iniciadas

Investigaciones a iniciarse

Tema, director, objetivo y descripción de no más de 200 palabras, año en que se iniciará y su relación con los items anteriores de este punto. 